\title{
SATISFAÇÃO EM RELACIONAMENTO B2B: INVESTIGACCÃO DA RECIPROCIDADE NA RELAÇÃO ENTRE MÉDICOS E PLANOS DE SAÚDE NO ESPÍRITO SANTO
}

\author{
SATISFACTION IN B2B RELATIONSHIP: RESEARCH OF \\ RECIPROCITY IN THE RELATION BETWEEN DOCTORS AND \\ HEALTH PLANS IN THE ESPÍRITO SANTO
}

Data de submissão: 30/11/2014 Aceite: 18/01/2016

André Sarmento Spalenza ${ }^{1}$

Felipe Andreão Ronchi²

Anderson Soncini Pelissari ${ }^{3}$

\section{RESUMO}

Para entender a satisfação em um ambiente B2B é necessário ter uma percepção sobre a troca de valores entre as partes envolvidas. Quando se fala sobre satisfação em relação a planos de saúde, ao realizar uma pesquisa nas bases de dados da CAPES, ANPAD e SCIELO, percebe-se que muitos estudos realizados se referem à satisfação do paciente perante o plano, mas poucos tratam da satisfação do médico, que também é cliente do plano, em uma relação business to business (B2B). Assim, o presente artigo analisa a relação entre os médicos e os planos de saúde no ES, e qual a percepção de valor na geração da satisfação por parte dos médicos. O estudo foi dividido em duas fases, a primeira, que foi um estudo qualitativo no formato de um grupo focal e por seguinte um estudo quantitativo, utilizando-se de questionário fechado. O questionário foi enviado por e-mail aos médicos inscritos no Conselho Regional de Medicina do Espirito Santo, totalizando 8.581 e foram respondidos por 236 profissionais. Sendo que, dessa quantidade, 230 eram credenciados em pelo menos um plano de saúde. O estudo empregou a Matriz Importância Desempenho para classificar a percepção dos médicos credenciados, além da análise dos GAP's, do teste T-Student e da correlação para a interpretação dos dados. Os GAP' s entre importância e desempenho encontrados em todos os itens demonstraram que o desempenho foi abaixo do esperado, porém, encontrou-se que de todos estes itens, o processos de autorizações para clientes é o que mais urgentemente precisa se adequar.

Palavras-chave: Satisfação, planos de saúde, matriz Importância Desempenho, GAP’s de qualidade.

\footnotetext{
1 Possui graduação e mestrado em Administração pela Universidade Federal do Espírito Santo, UFES, especialização em Administração de empresas pela Fundação Getúlio Vargas, FGV e doutorado em andamento em Administração Universidade Federal da Bahia, UFBA. Vitória. Espírito Santo. Brasil. E-mail: andrespalenza@gmail.com

2 Possui graduação em Ciências Econômicas pela Universidade Federal do Espírito Santo, UFES, graduação em andamento em Engenharia de Produção pela Fundação de Assistência e Educação, FAESA, especialização em Gestão Estratégica de Negócios pela Fundação Instituto Capixaba de Pesquisa em Contabilidade, Economia e Finanças, FUCAPE e mestrado em andamento em Administração pela Universidade Federal do Espírito Santo, UFES. Vitória. Espírito Santo. E-mail: felronchi@gmail.com

3 Possui graduação em Administração de Empresas, especialização em Recursos Humanos, especialização em Marketing pela Faculdade Espírito Santense de Administração, FAESA, mestrado em Engenharia de Produção pela Universidade Federal de Santa Catarina, UFSC e doutorado em Engenharia de Produção pela Universidade Metodista de Piracicaba, UNIMEP. Atualmente é Professor Nível C - Adjunto IV do Departamento de Administração da Universidade Federal do Espírito Santo, UFES. Professor do Programa de Pós-graduação em Administração - Mestrado e Doutorado - PPGADM, UFES. Vitória. Espírito Santo. Brasil. E-mail: asoncinipelissari@gmail.com
} 


\begin{abstract}
In order to understand the satisfaction in a B2B scenery, it is necessary to have an inside look at how the exchange of values between the sides happens. When talking about satisfaction with healthcare plans, given a research within the databases of CAPES, and ANPAD SCIELO, it is possible to conclude that many studies are related to patient satisfaction with the healthcare plans, but few deals with the satisfaction of doctors, who are also clients in this business to business relationship. Thus, this article analyzes the relationship between physicians and the healthcare plans in the ES, and their perception of value in generating satisfaction. This study was divided into two phases, the first, which was a qualitative study in a focus group format, followed by a quantitative study using a closed questionnaire. The questionnaire was sent via email to all physicians registered at the Regional Council of Medicine of Espírito Santo, in a total of 8581, with a return amount of 236 questionnaire. From that amount, 230 were accredited to at least one healthcare plan. The study employed the Importance-Performance Matrix to classify the perception of accredited doctors, and analysis of GAP's T-Student test and the correlation in data interpretation. GAP's between importance and performance found in all items showed that the performance was lower than expected, however, it was found that in all these items, the process of authorization for clients is what most urgently needs to adapt.
\end{abstract}

Keywords: Satisfaction, healthcare plans, importance-performance matrix, quality GAP's

\title{
1 INTRODUÇÃO
}

Os planos de saúde, no Brasil, constituem uma importante via de atendimento ao cidadão. Todo ano cresce o número de associados, e atualmente são 49 milhões de pessoas que se beneficiam com os planos de saúde, sendo mais de um milhão de pessoas somente no estado do Espirito Santo. Com o aumento da população beneficiada pelos planos, aumenta também o número de planos e médicos necessários para o atendimento (ANS, 2013).

Os pacientes são consumidores do plano de saúde, pois utilizam seus serviços por meio dos médicos, e os médicos também são consumidores, pois possuem com as operadoras de saúde uma relação Business to Business (B2B). Como aponta Prieto e Carvalho (2005), uma relação B2B ocorre na forma de associação entre duas partes, nenhuma delas é o consumidor final, mas, sim, oferece o produto ou serviço para outro cliente, como ocorre com os médicos em relação às operadoras.

Para entender a satisfação em um ambiente B2B é necessário ter uma percepção sobre como ocorre esta troca de valores entre as partes envolvidas. Em uma relação deste tipo, o vinculo entre as partes tende a ser de longa duração, e o valor esperado, em relação ao valor percebido, é um importante indicador que mede a satisfação neste campo (MORGAN; HUNT, 1994; WEITZ; BRADFORD, 1999; DAY, 2000; WALKER; NEELEY, 2004).

$O$ presente artigo traz um estudo a respeito da satisfação dos médicos em relação às operadoras de plano de saúde, em uma relação B2B, relações estas, que são consideradas como típicas de ambientes complexos (FRANCE; GROVER, 1992). Weick (1995) define um ambiente complexo como sendo aquele em que as relações são de difícil identificação e estão pouco articuladas. Este ambiente necessita de uma atenção diferenciada, por não funcionar como as organizações tradicionais. Na medição da satisfação, em relações complexas, um eficiente meio é a percepção de valores, entre o que é esperado pelo cliente e o que é recebido por ele, em uma análise da importância e do desempenho (MARTILLA; JAMES, 1977; PARASURAMAN; ZEITHAML; BERRY, 1985). Percepção esta que é comprovada no trabalho de Gianesi e Corrêa (2008).

Quando se fala da satisfação médica, em relação aos planos de saúde, inserida em um ambiente complexo (FRANCE; GROOVER, 1992), faz-se necessário entender o contexto atual do sistema de saúde e como os planos e os médicos se relacionam nele. $O$ sistema médico no Brasil é divi- 
dido, principalmente em três grandes partes, os médicos que trabalham para o SUS (Sistema Único de Saúde), os médicos que atendem em caráter particular e os que atendem por planos de saúde (ANS, 2013). Em relação ao atendimento realizado pelo SUS, como escreve Liberalesso (2011), poucas unidades conseguem manter um padrão de qualidade aceitável. O sistema que deveria atender a todos, com qualidade, acaba por não satisfazer o público, que encontra grandes filas, aparelhos muitas vezes em estado defasado, dentre outros. Médicos que vivem de clínicas particulares, segundo Liberalesso (2011) são raros, tal fato ocorre, pois o alto valor cobrado por uma única consulta restringe o público, que em sua maioria não está disposto a dispender desse recurso.

Tendo em vista a dificuldade de atendimento pelo SUS e a melhoria do padrão de vida de boa parte da população brasileira, bem como a popularização de alguns planos de saúde, segundo pesquisa realizada pela ANS (2013), grande parte da população está aderindo aos planos de saúde, por proporcionarem uma melhor qualidade de serviço, porém sem pagar um alto valor por ele. Os médicos então, em sua maioria, acompanham o mercado, ou trabalham para a rede SUS ou se associam aos planos de saúde (LIBERALESSO, 2011).

Silva (2003) salienta que os Prestadores de Serviços, inclusive os Médicos, por sua vez, atendidos nos seus interesses mais imediatos, notadamente pela progressiva escassez dos pacientes privados e pela crescente deterioração do relacionamento com a área pública (defasagem acentuada e crescente de preços, glosas, atrasos nos pagamentos, tetos físicos e financeiros incompatíveis com o volume dos serviços prestados, entre outros), não relutaram em compor uma associação forte e firme com as operadoras de planos de saúde. Liberalesso (2011) comenta que ao aderir a um plano de saúde, o médico se torna refém deste. $O$ baixo valor pago pelos planos é, segundo o autor supracitado, uma das principais reclamações, e este fator faz com que a qualidade do atendimento caia, pois, se se paga mal por cada consulta, o profissional é obrigado a realizar mais consultas em um único dia, realizando o serviço de forma não satisfatória.

Tendo em vista o embate, entre médicos e planos de saúde, o artigo propõe a seguinte questão: Quais são os principais elementos que contribuem para a satisfação do médico credenciado ao plano de saúde, e como o médico percebe o desempenho destes elementos pelo plano de saúde? Considerando a saúde como ambiente diferenciado, que também tem a necessidade de se medir a satisfação, e mais precisamente o elo existente entre os médicos e planos de saúde, propõe-se identificar os influenciadores de satisfação do médico, por meio de um grupo focal. Após a identificação destes itens, a importância e o desempenho dos mesmos para os médicos do Estado do Espirito Santo em relação aos planos de saúde. O objeto de estudo foram os médicos do banco de dados do Conselho Regional de Medicina do Espirito Santo (CRM-ES).

$\mathrm{O}$ artigo se justifica, pelo fato de que, quando se fala sobre planos de saúde, o principal objeto de estudo é sempre o paciente e não o médico. Com base em pesquisas nas bases de dados da ANPAD, SCIELO e CAPES, poucos estudos foram encontrados que apontavam a satisfação do médico em relação aos planos de saúde. Bem como os pacientes são clientes dos planos de saúde, assim, os médicos também são, em uma relação B2B, que necessita de uma investigação a respeito da satisfação, afim de que boas relações sejam construídas. $O$ artigo busca discutir inicialmente alguns elementos teóricos que auxiliaram na construção do estudo, bem como uma breve descrição do setor para o qual se propõe a pesquisa, preparando a posterior apresentação do método e dos resultados obtidos que fundamentaram a construção de um modelo de importância e desempenho (MARTILLA; JAMES, 1977).

O presente artigo se divide em quatro partes, sendo a primeira a revisão bibliográfica, que vai trazer autores que abordam a satisfação em um relacionamento B2B; marketing de relacionamento; importância e desempenho e a contextualização de sistema de saúde e dos planos 
de saúde no Brasil. Já na segunda parte, aborda-se sobre a metodologia, que tratou de explicar como foi feita a pesquisa e em que ela se baseou. Levando em conta o referencial abordado e a questão da satisfação, a pesquisa busca apontar o grau de importância dos pontos tratados e o grau de satisfação em relação a eles. A terceira parte analisou os resultados nos aspectos considerados mais relevantes. Por fim, a quarta parte apresentou as principais conclusões dos autores e a relevância que o trabalho teve.

\section{FUNDAMENTAÇÃO TEÓRICA 2.1 Satisfação em Relacionamento B2B}

Para medir a satisfação, Prieto e Carvalho (2005) falam do ambiente de negócios como sendo B2C (business to consumer), onde se mede a satisfação do cliente na condição de consumidor, e B2B (business to business), que mede a satisfação entre duas empresas, em uma relação de colaboração. A respeito do relacionamento B2B, o estudo realizado por Lacerda e Mendonça (2010) aponta que este campo tem ganhado destaque nas publicações acadêmicas desde 2002, em que, anteriormente a essa data, não era muito considerado quando se falava a respeito de satisfação.

No tipo de relação B2B, Walker e Neeley (2004) e Gummerson (1996) salientam o interesse das partes em manter relações duradouras, pois a perda de um cliente neste tipo de relação implica em insucesso, uma vez que as trocas realizadas em ambientes B2B geralmente são de grande valor agregado, essa ótica da relação em longo prazo, para Gummerson (1996) acontece também em uma relação B2C, porém se dá de maneira mais efetiva em um mercado B2B, o que também é indicado nos estudos de Olkoski et. al (2009) e Lacerda e Medonça (2010). O alto valor agregado ocorre, pois segundo Day (2000) e confirmado por Prieto e Carvalho (2005) os processos em uma relação B2B geralmente são feitos em vários passos, que envolvem negociações.

Walker e Neeley (2004), bem como Day (2000), ao falar sobre o interesse em manter um bom relacionamento, em um acordo B2B tratam a satisfação baseada em um processo de troca de valores, onde há a percepção de um valor que é dado e outro que é recebido. Assim, os autores abordam sobre o relacionamento baseado em valores esperados, fundamental para uma relação ao longo prazo, necessária para uma boa parceria B2B.

Ao entender troca de valores e bom relacionamento, como elementos essenciais, para uma boa parceria em um ambiente business to business, Walker e Neeley (2004) relatam sobre um marketing voltado para o relacionamento, que é definido por Morgan e Hunt (1994) como sendo primordial para aperfeiçoar, manter e estabelecer relações duradouras, de forma a gerar lucro com a realização dos objetivos das partes. O relacionamento gerado, em geral é de longo prazo, o que garante a vantagem competitiva (MORGAN; HUNT, 1994; WALKER; NEELEY, 2004; CHANG et al, 2012).

Para gerar valor para o cliente e alcançar a vantagem competitiva, Chang et al (2012) apontam que é necessário entender o perfil do seu cliente, para trabalhar um relacionamento de forma especifica. Os autores ainda apontam, que do mesmo modo em que o marketing de relacionamento, em uma situação direta com o cliente, tem de se preocupar com as particularidades de cada um aos quais atende em um ambiente B2B, também se deve atentar para as diferenças de cada um com quem se relaciona, e em cima das particularidades dos clientes, trabalhar as variáveis consideradas importantes. Essas variáveis, que serão consideradas importantes é que geram valor e aumentam a vantagem competitiva (CHANG et al, 2012), tal fato é comprovado pelo estudo realizado por Moran et al (2013), sendo também identificado no estudo dos autores, que cada variável possui um peso diferente na definição da geração de valor. 
Para a geração de valor e vantagem competitiva em um mercado B2B, a criação de valor para o cliente é importante, e propicia a construção de parcerias para todas as partes envolvidas neste relacionamento (WEITZ; BRADFORD, 1999; DAY, 2000; OLKOSKI et. al, 2009). Para que a construção de valor seja construída de forma efetiva, Day (2000) afirma a importância da comunicação entre os envolvidos, e a importância da clareza quando a empresa fornecedora do serviço ou produto passa as informações para os que adquirem esses produtos ou serviços, bem como a clareza de quem adquire para com os fornecedores dos produtos ou serviços.

Morgan e Hunt (1994) afirmam em seu estudo que, em um ambiente business to business, existem vários tipos de relacionamentos, que variam do tipo da área empresarial trabalhada e devem ser trabalhadas de forma específica. Na área de saúde, principalmente na relação entre médico e plano de saúde, conforme afirmam France e Grover (1992) as relações assumem aspectos complexos, pela intangibilidade dos serviços e complexidades das relações, onde ao mesmo tempo, que o médico depende do plano de saúde, este tem completa liberdade em relação a ele.

Tendo em vista a importância do relacionamento em um ambiente, business to business, para manter a satisfação (WALKER; NEELEY, 2004), baseado na troca de valores (MORGAN; HUNT, 1994) e levando em consideração a complexidade do setor, em que os planos de saúde se encontram (FRANCE; GROVER, 1992), um estudo a respeito da satisfação dos médicos em relação aos planos de saúde deve estar atento às estratégias de relacionamento, definidas pelo marketing relacional.

\subsection{O marketing de relacionamento.}

Gummerson (1996) aponta que o marketing de relacionamento ganha destaque no começo da década de noventa e contempla, fundamentalmente, três premissas: relacionamento, rede e interação. Neste sentido, Curi (2007) destaca que o marketing de relacionamento trata, mais do que somente os clientes, mas uma rede por completo, em suas interações. O marketing de relacionamento então é um sistema complexo, que engloba diversos aspectos em diversos níveis. A disciplina contempla todos os envolvidos para se chegar a um consumidor final, e todas as relações existentes neste processo (GUMMERSON, 1996; DAY, 2000; CURI, 2007). O marketing de relacionamento é então, como aponta Gomes et al. (2012) fundamental para que a organização entenda seus clientes e alcance a vantagem competitiva.

Segundo Day (2000), as relações trabalhadas no marketing relacional ganham destaque quando se fala em um relacionamento complexo, como ocorre em um ambiente $B 2 B$, onde as relações existentes são muito mais difíceis de serem feitas e mantidas, do que em um relacionamento B2C, pois quando se fala de um relacionamento corporativo, a criação de valor é mais dificilmente alcançada.

Quando se fala em marketing de relacionamento, McKenna (1996) assume que as relações são a chave a serem consideradas para esse tipo de abordagem. $O$ autor aborda a importância do relacionamento duradouro levando em consideração um ambiente com mercados altamente instáveis e imprevisíveis, o que também é identificado em Gomes et al. (2012), sendo o relacionamento um suporte para se enfrentar as dificuldades impostas. Mckenna (1996) aponta o marketing de relacionamento como voltado para a criação de mercados, se baseando no conhecimento e na experiência definindo os recursos, e não a abordagem de desenvolvimento de produtos.

Para que ocorra a criação de mercados, baseados no conhecimento e na experiência (MCKENNA, 1996), Henning-thurau e Klee (1997) falam da qualidade do relacionamento, que deve ser adequada, no sentido de satisfazer o cliente, oferecendo a ele, uma boa experiência, que gera confiança, o que leva a satisfação. O Marketing de relacionamento, se baseia principalmente na confiança para a geração de satisfação, confiança, que é definida por Palmatier et al (2006) como a 
crença de que um provedor de um determinado serviço o realizará de maneira íntegra e credível. Morgan e Hunt (1994), ainda trazem a questão da prestatividade, na geração da confiança, pois para os autores, de nada adianta a integridade e a segurança no serviço, se não houver a prestatividade.

O marketing de relacionamento então traz a qualidade do serviço, juntamente com a confiança e a satisfação na geração de um bom relacionamento, relacionamento este que é essencial para a manutenção dos clientes e a geração de vantagem competitiva. Quando há um bom relacionamento, firma-se um compromisso de trabalho, e é criado um elo, entre empresa e cliente (MORGAN; HUNT, 1994; HENNING-THURAU; KLEE, 1997; PALMATIER et al, 2006).

Morgan e Hunt (1994) apontam que a confiança é um fator crítico na manutenção de um bom relacionamento, que propicia uma troca entre os lados no longo-prazo e não apenas no curto, porém segundo os mesmos autores, e também observado por Pinho (2013) esse fator deve vir acompanhado de outros, conforme o contexto em que se trabalha, pois para cada contexto, o relacionamento segue regras específicas, como o exemplo citado por Pinho (2013) do relacionamento baseado em status social.

O estudo realizado por Rozzet e Demo (2010) comprova a relação positiva entre confiança e satisfação na geração de um bom relacionamento. As autoras utilizaram a ERC (Escala de Relacionamento com Clientes) em um ambiente B2B, e comprovaram que para ter um bom relacionamento é necessário a confiança e a satisfação, o que é apontado por Henning-thauru e Klee, 1997, Morgan e Hunt, 1994 e Palmatier et al, 2006.

Por meio de estudos, realizados por Palmatier et al (2006) e também por Rozzet e Demo (2010), percebeu-se que o Marketing de relacionamento está muito ligado a organizações que possuem relações complexas, como ocorre na área da saúde (FRANCE; GROOVER, 1992). O estudo de Palmatier et al (2006), bem como o de Rozzet e Demo (2010) foram realizados através de análises a respeito dos fatores que influenciam eficácia do marketing de relacionamento em um ambiente B2B. Sendo que em Palmatier et al (2006) se realiza uma meta-análise e em Rozzet e Demo (2010) se utiliza uma escala, para medir quantitativamente a importância do marketing de relacionamento. Olkoski et al. (2009) apontam a importância de se medir o relacionamento de forma quantitativa, pois, através destas análises é possível se repensar no que se investe, em relação ao que é considerado, de fato, importante para se alcançar um relacionamento positivo.

\subsection{Importância e Desempenho}

Quando se fala em medir satisfação em relações complexas, levando em consideração o relacionamento, é importante medir o que é considerado importante e o que é desempenhado em relação aos itens considerados importantes, a fim de que seja estabelecida uma relação de ganha-ganha (MARTILLA; JAMES, 1977; PARASURAMAN; ZEITHAML; BERRY, 1985).

Martilla e James (1977) introduziram o conceito de Matriz de Importância e Desempenho, que permite a empresa ter uma visão sobre os atributos considerados importantes por seus clientes e como a empresa está oferecendo estes atributos, se os clientes estavam satisfeitos ou não. Utilizando essa Matriz, Gianesi e Corrêa (2008) apontam que é possível avaliar a importância e o desempenho da empresa em relação ao atendimento das expectativas para cada um dos elementos testados.

Para se identificar a importância, utiliza-se uma escala numérica direta ou uma regressão linear e a importância é medida por meio do coeficiente linear do desempenho do serviço e da satisfação geral. O desempenho é dado levando em consideração a concorrência e as percepções gerais do mercado ou ao desempenho que se espera em determinado setor (GARVER, 2003). 
A Matriz (importância e desempenho) pode ser utilizada de forma diferente, dependendo do autor, porém, mantêm-se as suposições de que o desempenho está linearmente ligado à satisfação e que a importância e o desempenho são variáveis independentes (SLACK; CHAMBERS; JOHNSTON, 2009). Para medir estas variáveis a Matriz é dividida em quatro quadrantes, conforme a figura 1 :

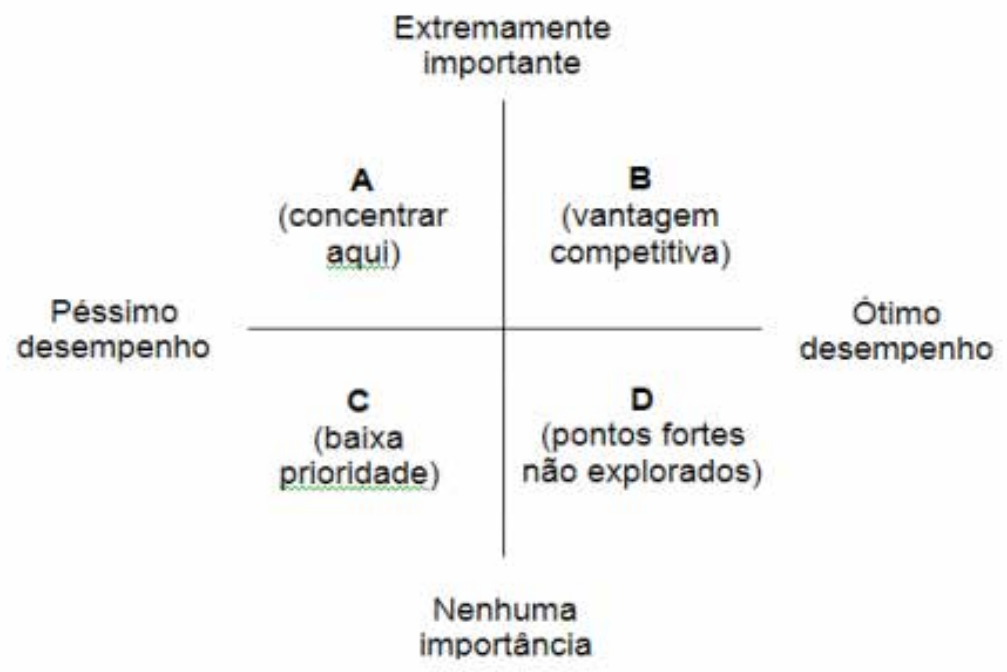

Figura 1: Matriz Importância e Desempenho Fonte: Tonge e Moore, 2007.

Os atributos localizados no quadrante $A$, indicam baixo desempenho e que se deve adequar urgentemente estes itens. Os itens localizados no quadrante $B$ indicam grande vantagem competitiva, e devem ser mantidos. $O$ quadrante $C$ representa itens que não tem bom desempenho, porém não possuem muita importância para o cliente. $O$ quadrante $D$ representa desperdício de recursos, pois o item possui grande desempenho, porém pouca importância (TONGE; MOORE, 2007). Para cada item, é proposta uma pontuação, baseada na média de todas as respostas de cada item medido. Esta pontuação permite que seja medida a importância e o desempenho, enquadrando cada atributo na Matriz (MARTILLA; JAMES, 1977). Para dividir cada quadrante, a literatura não traz um consenso sobre onde deve começar e terminar cada uma das quatro zonas, autores criam divisões diferentes, porém é necessário que, depois de estabelecida a zona, ela seja mantida em toda a pesquisa (SLACK; CHAMBERS; JOHNSTON, 2009).

Para a análise da importância e desempenho, Gianesi e Corrêa (2008), baseado no trabalho de Parasuraman, Zeithaml e Berry $(1985,1988,1990)$ utilizam além da Matriz de Importância e Desempenho (MARTILLA; JAMES, 1977) a análise de GAPs (lacunas) de qualidade que são segundo Slack; Chambers; Johnston (2009), estudos complementares.

A análise de GAPs é a percepção da diferença entre o que é considerado importante e como isso é realizado pela empresa. As relações que possuem um GAP (Lacuna) entre o que é considerado importante e o que é desempenhado indicam uma falha de qualidade, sendo estas falhas mais ou menos importantes levando em consideração o tamanho do GAP entre a importância e desempenho (PARASURAMAN; ZEITHAML; BERRY, 1988).

A análise de GAPs, proposta por Parasuraman, Zeithaml e Berry $(1985,1988)$ recebeu criticas de autores como Carman (1990) e Cronin Jr e Taylor (1992), que indicam que essa análise não possui consistência, e por isso não pode ser utilizada como única ferramenta na realização de um estudo sobre importância e desempenho, mas sim como suporte para alguma outra fer- 
ramenta de pesquisa, afim de que o resultado encontrado tenha consistência. Por isso, para este trabalho, utilizou-se esta análise, juntamente com a matriz importância e desempenho.

A análise de importância e desempenho possui como principal função, segundo Tontini e Sant'ana (2007), auxiliar os gestores das empresas na visualização dos produtos ou serviços oferecidos pela empresa, possibilitando à empresa a verificação de quais características e aspectos ofertados poderiam ser aperfeiçoados, permitindo assim à empresa uma ampliação de sua competitividade no mercado.

\section{CONTEXTUALIZAÇÃO DO SISTEMA DE SAÚDE E DOS PLANOS DE SAÚDE NO BRASIL.}

A atual fase dos sistemas de saúde caracteriza-se pela introdução de mecanismos de mercado na provisão dos serviços, pelas proposições de renúncia do Estado à responsabilidade por essas funções e pelo fortalecimento da participação da oferta e das despesas privadas em saúde. Com o crescimento do setor privado, na assistência à saúde, com destaque especial ao setor supletivo, o Estado que acabou por se afastar, em parte, da prestação dos serviços, passou a ganhar importância na regulamentação do setor privado (SATO, 2007).

Os planos de saúde constituem no Brasil uma importante via de atendimento ao cidadão, tendo em vista que atualmente 49 milhões de pessoas estão associadas a algum tipo de plano, conforme informações da Agência Nacional de Saúde Suplementar (doravante ANS). Em relação ao Espírito Santo, objeto de estudo deste artigo, o Estado possuí mais de um milhão de beneficiários de planos privados de saúde (ANS, 2013).

A regulação desse setor se consolidou a partir de 1998, com a Lei no 9.656/1998. É justamente nesse contexto, de regulamentação, que nasceu a Agência Nacional de Saúde Suplementar (ANS) em 2000. A ANS tem como função fiscalizar e regulamentar os contratos entre os usuários e as operadoras de saúde. Entretanto, o impacto não se restringe a esses dois atores. Todos os prestadores, como hospitais, laboratórios, profissionais, entre outros, são influenciados pelas medidas tomadas pela ANS (LEAL; MATOS, 2009).

Ao longo dos últimos anos, o número de beneficiários de planos de saúde cresceu significativamente, apresentando uma distinção marcante quanto ao tipo de contratação, que pode ser individual/familiar (doravante individual) ou coletiva, esta última, com participação e crescimento mais significativos, totalizando mais de 38 milhões de beneficiários $(78,7 \%$ em relação ao total de beneficiários) (ANS, 2013). No Espírito Santo, a contratação coletiva possui 971 mil beneficiários (83,92\% em relação ao total de beneficiários). A contratação coletiva, em geral, está relacionada ao mercado de trabalho, pelo fato de ser o segmento no qual os contratantes são pessoas jurídicas, diferentemente do mercado individual, no qual os contratantes são pessoas físicas.

\section{METODOLOGIA}

A pesquisa de Marketing constitui em estudar como ocorre o impacto de determinadas variáveis sobre determinado assunto, bem como, o modelo que essas mesmas variáveis influenciam este assunto. Para Malhotra (2006), ao se realizar uma pesquisa de marketing, é necessário, primeiro reconhecer um problema. Após o reconhecimento da questão norteadora se efetua um planejamento, que envolve a definição dos aspectos relacionados à aplicação da pesquisa, bem como o local que será aplicado, regras e recursos. Definido o planejamento se parte para a fase da execução, que é a coleta dos dados. Após serem coletados, os dados, faz-se uma análise e mede a relevância deles, bem como se tira as principais conclusões. 
O problema reconhecido, neste artigo, foi apontar quais os principais elementos que contribuem para a satisfação do médico que se credencia a um plano de saúde, assim há uma relação B2B, em que o médico presta serviço aos Planos de Saúde e os Planos de Saúde pagam os médicos, pelos serviços prestados aos pacientes. Para responder ao problema proposto, foi realizado um grupo focal, que será mais bem descrito posteriormente, com a presença de seis médicos. Neste grupo, os médicos deram suas opiniões a respeito de questões que eles achavam importantes para a resposta do problema proposto. Baseado na opinião dos médicos, presentes no grupo, e nos achados de Silva (2003) e Liberalesso (2011) confeccionou-se um questionário, no qual foi enviado para todos os 8.581 médicos do banco de dados do CRM-ES, onde não há registro de quais e quantos médicos fazem parte de algum plano de saúde. Os 230 questionários respondidos, de médicos credenciados a pelo menos um (1) plano de saúde, de forma válida foram analisados e, a partir disso, foram tirados os aspectos mais importantes, que serão descritos detalhadamente neste artigo.

Em uma pesquisa de satisfação dos prestadores de serviços, em relação à empresa contratante, a geração dos indicadores de satisfação reveste-se de extrema importância, uma vez que é deles que resulta a validade deste tipo de pesquisa. Na prática isso significa dizer que o prestador de serviço precisará expressar estados de satisfação (e/ou insatisfação) em relação a elementos por ele valorizados nas suas relações com a empresa. De nada adiantará medir estados de satisfação e/ou insatisfação em relação a elementos para os quais os prestadores de serviços, neste caso os médicos, se mostrarem indiferentes (ROSSI; SLONGO, 1998).

Segundo Rossi e Slongo (1998) errar na definição dos indicadores de satisfação corresponde a errar na essência da pesquisa. Por mais perfeito que seja o plano de pesquisa nos seus aspectos de amostragem, procedimentos de coleta, análise e interpretação de resultados, ele sucumbirá se sua base de sustentação estiver fragilizada pela ausência de bons indicadores de satisfação, gerados com base científica. Embora possa existir forte propensão a fazê-lo, é bom lembrar, também, que a empresa não deve construir o instrumento da pesquisa para medição da satisfação de seus prestadores de serviços em cima de indicadores gerados exclusivamente pelos próprios executivos, ou simplesmente retirados da literatura especializada. Por mais experientes que sejam os executivos, ou por mais profunda e atualizada que seja a literatura, será necessário dotar a pesquisa de características específicas, devidamente sintonizadas com o perfil do prestador de serviço cujo grau de satisfação se pretende medir. Trata-se, portanto, de customizar a pesquisa.

Desta forma, o presente estudo apresenta duas fases distintas e bem demarcadas em termos de tempo e de método: a primeira, de natureza exploratória e qualitativa, conduzida com o propósito de gerar os indicadores de satisfação, que fornecerão a base para a elaboração do instrumento de coleta de dados da segunda fase; a segunda, de natureza descritiva e quantitativa, destinada a medir o grau de satisfação dos clientes. A seguir são apresentadas e comentadas estas duas fases.

\subsection{Fase 01 - Estudo Exploratório Qualitativo.}

Para Bogdan e Biklen (1994), a pesquisa qualitativa trabalha com dados subjetivos, como crenças, valores e opiniões. Neste tipo de pesquisa, considera-se a história do entrevistado e suas impressões acerca do assunto abordado. Richardson (2007) afirma que este tipo de estudo é ideal quando se quer entender a natureza de um fenômeno social observado. $O$ método qualitativo é utilizado quando os fenômenos observados são de natureza complexa e envolvendo aspectos subjetivos, o que torna inviável a quantificação deles para serem compreendidos. $O$ estudo qualitativo observa processos dinâmicos de grupos e é muito útil a utilização deste método quando não se 
possui muitas informações acerca do que se pretende estudar (RICHARDSON, 2007). A abordagem exploratória busca o descobrimento de práticas com base em novas descobertas, este tipo de análise é feito quando não se possui hipóteses anteriormente formuladas, o que possibilita ao pesquisador descobrir elementos que não eram esperados (BOGDAN; BIKLEN, 1994; RICHARDSON, 2007).

Para a realização da fase um, onde os principais pontos seriam levantados, a fim de se confeccionar um questionário que respondesse o que se propôs, realizou-se um grupo focal. Foi escolhida essa técnica, pois segundo Dall'Agnol e Trench (1999), ela é bastante utilizada nas áreas de educação e saúde. A vantagem dessa técnica se dá pelo fato de ideias poderem emergir em meio às discussões, permitindo assim a averiguação de determinado assunto em caráter mais profundo. Ainda segundo as autoras, o grupo focal permite a reflexão dos pesquisadores a cerca das próprias concepções. Flick (2004) conceitua o grupo focal como sendo, na realidade, uma entrevista e não uma discussão. Nesta entrevista, realizada em grupo, cada um dos membros coloca sua opinião, o que gera um debate, que é moderado e incentivado pelo entrevistador.

O grupo focal, realizado, contou com a presença de seis médicos, de especialidades diferentes, e durou em torno de quarenta minutos. A literatura indica que o grupo focal deve contar com, no mínimo seis, e no máximo quinze membros, e deve possuir duração de trinta minutos até duas horas (DALL'AGNOL; TRENCH, 1999; FLICK, 2004).

Desta forma, primeiramente, com base no referencial utilizado no presente estudo, foram selecionados os indicadores que tratavam sobre satisfação dos médicos em relação aos planos de saúde. Pela escassez de estudos e a inexistência de um questionário específico, foi necessária a elaboração do grupo focal, cujo objetivo foi obter informações relevantes para incluir no questionário.

Um facilitador apresentou a visão geral e os objetivos da discussão, sendo estes os indicadores que influenciam a satisfação dos médicos em relação às operadoras de plano de saúde. Posteriormente, foi realizada uma discussão em cima dos itens embasados na literatura, incentivando as opiniões dos participantes, de forma a avaliarem se estes realmente influenciam na satisfação. Além disso, foi sugerido para que os participantes apresentassem outros fatores que influenciam essa relação. Por fim, o facilitador, resumiu as informações contidas na discussão, confirmadas pelos demais participantes.

\subsection{Fase 02 - Estudo Descrito Quantitativo.}

Com base nos resultados obtidos na pesquisa qualitativa, iniciou-se a segunda fase da pesquisa, denominada de pesquisa quantitativa descritiva. Esta fase permitiu obter as informações necessárias, a continuidade do projeto e ao efetivo alcance dos objetivos propostos. O método quantitativo descritivo é constituído pela utilização de técnicas estatísticas, desde as mais simples, até as mais complexas, para que seja feita a coleta dos dados e a análise destes. Este tipo de método permite precisão na análise dos dados e abre pouco espaço para distorções. O método quantitativo é principalmente utilizado quando se realiza um estudo descritivo, estudo este que se caracteriza por identificar e correlacionar variáveis, dependentes ou independentes. Este tipo se caracteriza por fazer uma classificação, interpretação e explicação de determinado fato (RICHARDSON, 2007).

O estudo quantitativo descritivo permite que seja percebido um nexo causal entre conceitos e permite que este nexo percebido, seja levado para além dos limites da pesquisa, ao se estudar outras situações que estão inseridas em contextos semelhantes e possuem características semelhantes as da população estudada, apresentando assim, uma declaração geral sobre o fenômeno estudado (SMITH, 1983). No estudo quantitativo, a pesquisa de campo é guiada, primeiramente por conceitos estruturados. Após a estruturação, desses conceitos, são formuladas hipóteses, que são levadas a campo, a fim de identificar a sua veracidade ou não. Os dados colhidos mostram informações quantificadas, que permitem identificar a ocorrência ou não da hipótese proposta (POPPER, 1972). 
Nesta fase, foi utilizada a pesquisa quantitativa, tendo os questionários enviados via banco de dados do CRM-ES. No caso da pesquisa aplicada, a população em análise são os médicos credenciados no mínimo a um plano de saúde. Pelo o fato do CRM-ES não dispor da relação de médicos credenciados e não credenciados a planos de saúde, foi-se necessário enviar o questionário para todos os médicos inscritos no Conselho, justificando o envio para todos. Para a pesquisa, utilizou-se a aplicação de um questionário, que segundo Smith (1983), é uma das técnicas mais utilizadas e eficientes para a coleta quantitativa em uma investigação social. Os questionários foram enviados para os 8.581 médicos atualmente cadastrados no CRM-ES, sendo estes compostos por credenciados e não credenciados a planos de saúde. De todos os questionários enviados, obteve-se resposta de 236 médicos, sendo estes, 230 credenciados, os outros seis questionários não considerados eram de médicos não credenciados a nenhum plano de saúde.

Como os questionários foram enviados para todos os médicos cadastrados, trabalhou-se com toda a população pretendida. Segundo Levine et al (2012), enviando-se o questionário para toda uma população, todos tem igual oportunidade de responder, evitando assim o viés de pesquisa, que é caracterizado por tendências que o entrevistador tem de escolher um elemento dentre todos os possíveis.

\subsubsection{Da elaboração do questionário}

O questionário elaborado buscou medir a importância e o desempenho, segundo a Matriz de Martilla e James (1977). A relação final de atributos foi operacionalizada por meio de uma escala intervalar, do tipo Likert, de sete pontos (CHURCHILL Jr., 1995; HAIR; BUSH; ORTINAU, 2000; MALHOTRA, 2006), apresentando em seus extremos (1) Totalmente Insatisfeito e (7) Totalmente Satisfeito. Já o termo intermediário (4) subentende-se como Neutro. A escala do tipo Likert, exige que os entrevistados indiquem um grau de sua percepção no que tange a cada afirmação ou pergunta, facilitando o entendimento dos respondentes e o tratamento das repostas (MALHOTRA, 2006).

$O$ instrumento de coleta de dados, ou questionário de pesquisa, diz respeito a uma técnica estruturada, que consiste em um conjunto de indagações que devem ser respondidas pelo entrevistado, de forma padronizada. Para a elaboração deste questionário, utilizou-se a relação de atributos resultante da fase exploratória. Assim que estruturado, o instrumento de coleta de dados foi feita a validação de conteúdo, com o objetivo de medir se o que foi perguntado, realmente mede o que se quer estudar (KINNEAR; TAYLOR, 1996). A validação do conteúdo consiste em uma avaliação subjetiva da representação dos aspectos estudados (MALHOTRA, 2006).

A metodologia estabelecida para identificar as relações entre determinantes e os atributos mais importantes de satisfação, do médico em relação ao plano de saúde, envolve levantamentos junto aos médicos, com base na matriz de importância e desempenho (MARTILLA; JAMES, 1977) para a identificação da satisfação de cada item. Assim, a confirmação de expectativas é resultado da comparação entre as expectativas prévias e o desempenho real do serviço (OLIVER, 1980; CHURCHILL; SURPRENANT, 1982).

Os determinantes contemplados para a modelagem da satisfação, dos médicos em relação aos planos de saúde são: Imagem do(s) plano(s) de saúde no mercado; Canal de comunicação com o(s) plano(s) de saúde; Processos de autorizações para clientes; Auditoria médica; Recursos de glosa nas contas médicas; Remuneração; Cumprimento de prazo de pagamento; e Rede de prestadores de serviços (laboratórios, clínicas, hospitais).

Desta forma, a aplicação da pesquisa é feita por meio de um questionário fechado, contendo oito questões, que objetiva avaliar as relações entre importância e satisfação do médico, frente a cada um destes itens. Além disso, no fim do questionário é realizada uma pergunta em relação à satisfação dos planos de saúde em geral, como visto em Garver (2003). 
Para obter a caracterização dos médicos entrevistados, foram realizadas sete perguntas que diz respeito aos seguintes itens: Gênero; Faixa etária; Locais de trabalho; Tempo de credenciamento; Quantidade de planos de saúde credenciados; Porcentagem da receita do(s) plano(s) de saúde em relação à receita total do médico e; Quais os planos o médico é credenciado (Opcional).

\subsubsection{Coleta dos dados}

Os dados foram coletados, na fase 01 , no mês de junho de 2014, na forma de um grupo focal, conforme indicam Dall'agnol; Trench (1999) e Flick (2004), contando com a participação de seis médicos, com a duração de aproximadamente 40 minutos. Com as informações contidas no grupo focal, e também baseado na literatura, foi possível confeccionar um questionário.

Na fase 02 da pesquisa, ocorrida no período de julho de 2014, os questionários foram enviados via banco de dados do CRM-ES para todos os médicos cadastrados, num total de 8.581 . No caso da pesquisa aplicada, como o banco de dados do CRM-ES contemplam todos os médicos, os cadastrados e não cadastrados a planos de saúde, sendo a população pretendida, somente os médicos cadastrados, foi necessário uma pergunta inicial, certificando que o respondente era cadastrado pelo menos a um plano de saúde.

Dos 8.581 questionários enviados, obtivemos a resposta de 236 médicos, sendo que, desse número, 230 , confirmaram serem cadastrados a pelo menos um plano de saúde, e seis, do total de respondentes, responderam ao questionário sem fazer parte de nenhum plano. Os respondentes não cadastrados a planos de saúde foram retirados do banco de dados por não atenderem ao pré-requisito de pertencer pelo menos a um plano de saúde.

\section{TRATAMENTO E ANÁLISE DOS DADOS 5.2. Fase 02 - Estudo Descrito Quantitativo.}

Para a criação do questionário, foi utilizado o Google Docs, e para não haver dados perdidos, o respondente só poderia enviar o questionário se todas as perguntas fossem respondidas. Para a tabulação dos dados, bem como para o cálculo das variáveis, foram utilizadas planilhas do Excel.

Buscando uma categorização da amostra, observa-se que do total de 234 , foram extraídos apenas os respondentes que possuíam credenciamento com pelo menos um plano de saúde. Refazendo um novo total de 230 respondentes. Desses 230 participantes, $82(35,7 \%)$ são do sexo feminino e 148 (64,3\%) são do sexo masculino. A maioria dos entrevistados está na faixa etária de 50 a 59 anos, representados por $29,1 \%$ da amostra, seguidos por $28,7 \%$ da faixa entre 60 a 69 anos, $24,3 \%$ da faixa entre 40 e 49 anos e 16,1\% entre 30 e 39 anos. Ademais, os respondentes com idade igual ou menor que 29 anos e a faixa entre 70 a 79 anos representaram apenas $0,9 \%$ da amostra, cada uma.

No que se refere ao local, que os médicos atuam com credenciamento de plano de saúde, tem-se que dos 230 respondentes, 122 (53\%) utilizam o credenciamento somente em consultórios/clínicas, 15 (6,5\%) somente em hospitais e por fim 93 (40,4\%) em ambos. Quanto ao tempo que o médico possui de credenciamento junto ao plano de saúde, tem-se os seguintes resultados com base nos 230 respondentes: $54(23,5 \%)$ de 18 a 23 anos, $43(18,7 \%)$ de 24 a 29 anos, $41(17,8 \%)$ de 12 a 17 anos, $36(15,7 \%)$ de 6 a 11 anos, 33 (14,3\%) de 30 ou mais anos, 21 $(9,1)$ de 5 anos ou menos e $2(0,9 \%)$ não sabiam. Em relação a quantidade de planos de saúde que os médicos possuem, os resultados mostraram que $37(16,1 \%)$ possui apenas um plano de saúde, $28(12,2 \%)$ dois planos e $165(71,7 \%)$ possuem 3 ou mais. 
Por fim, a respeito da porcentagem da receita dos planos de saúde frente a receita total do médico, observou-se que dos 230 médicos, 46 (20\%) responderam que a receita com o plano de saúde corresponde em até $25 \%$ de sua receita total. Adicionalmente, tem-se $43(18,7 \%)$ dos médicos com $26 \%$ a $50 \%$ da receita total, $57(24,8 \%)$ com $51 \%$ a $75 \%$ da receita total e $84(36,5 \%)$, correspondendo mais do que $75 \%$ da receita total. Desta forma, observa-se que $61,3 \%$ dos respondentes possuem pelo menos $50 \%$ da sua receita referente aos planos de saúde, corroborando com a afirmação de Liberalesso (2011) que comenta que ao aderir a um plano de saúde, o médico se torna refém deste.

Após a categorização dos respondentes, foram identificadas as médias de cada item, os gaps entre a importância e o desempenho, os desvios padrões, p-valor, a correlação e aplicada a matriz Importância e Desempenho para que fossem analisadas, conforme a tabela 1.

Realizando uma análise de Importância entre os itens medidos, para medir a satisfação geral com os planos de saúde, observa-se uma média de 5,27 entre os elementos. Os itens que obtiveram importância a cima da média foram o canal de comunicação para com o plano de saúde $(5,41)$, o processo de autorização para os clientes $(5,32)$, o cumprimento de prazo de pagamento $(5,92)$, e a rede de prestadores de serviço, como laboratórios, clínicas e hospitais $(5,75)$. Dentre os itens abaixo da média, obtivemos a imagem do plano de saúde para os clientes $(5,06)$, a auditoria médica $(4,77)$, recurso de glosa nas contas médicas $(4,98)$ e a remuneração $(4,89)$. A remuneração, indicada por Liberalesso (2011), como sendo considerada de extrema importância na medida da satisfação dos médicos, não foi percebida na realidade capixaba.

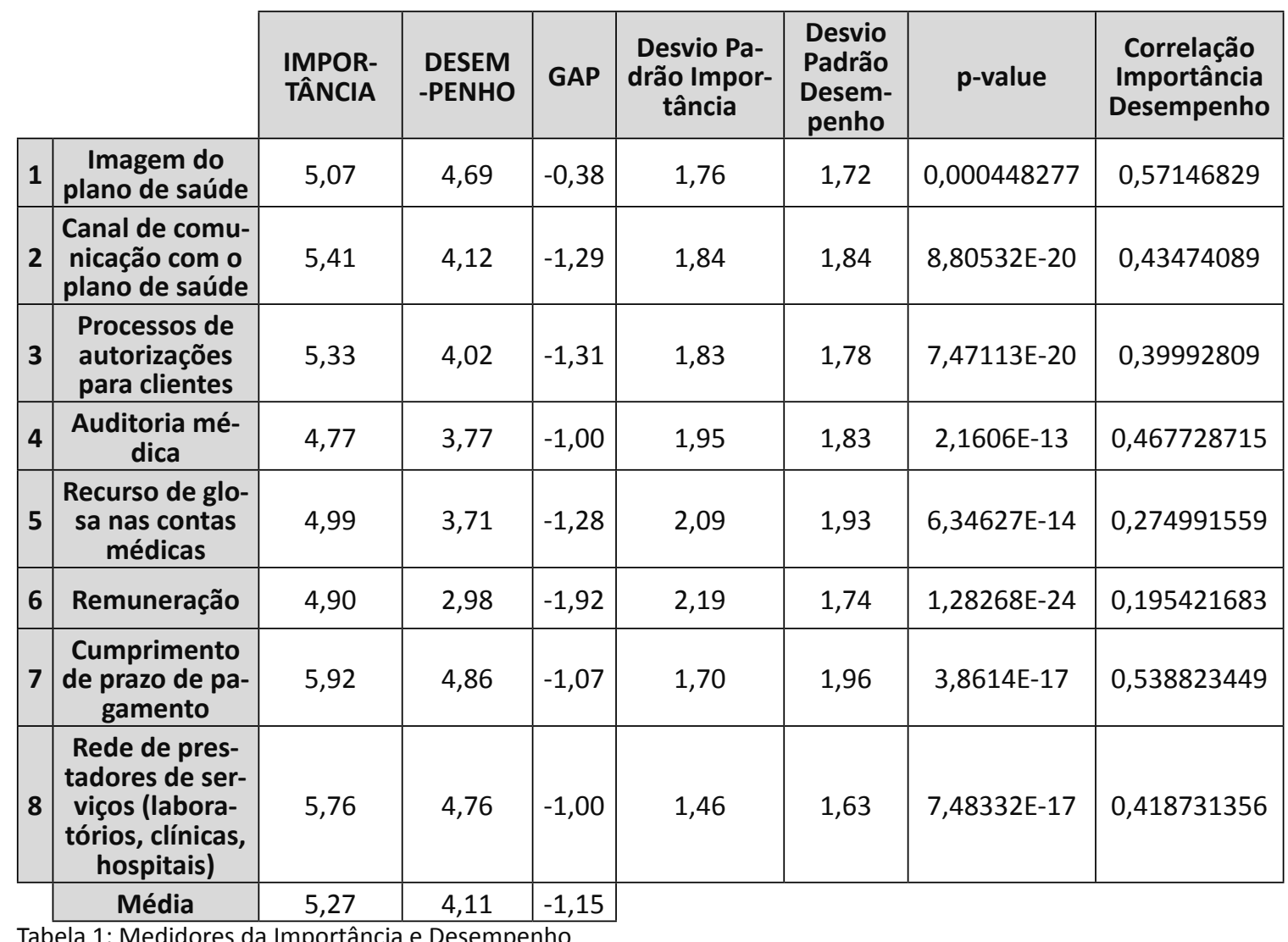

Fonte: Dados da pesquisa (2014) 
Quando analisado o desempenho, observa-se uma média geral de 4,11. Os itens demonstrados como os de desempenho acima da média foram: a imagem do plano de saúde $(4,69)$, canal de comunicação com o plano de saúde $(4,12)$, cumprimento do prazo de pagamento $(4,85)$, e a rede de prestadores de serviço $(4,76)$. Os itens identificados como abaixo da média do desempenho foram: processo de autorização para clientes $(4,01)$; auditoria médica $(3,76)$; recurso de glosa nas contas médicas $(3,70)$ e; a Remuneração $(2,97)$.

Foi identificado que, em todos os itens, o desempenho atingiu média menor do que a importância, como é apontado no gráfico 1, abaixo. Isto quer dizer que o desempenho dos planos de saúde está abaixo das expectativas dos médicos credenciados em todos os fatores pesquisados, o que vai ao encontro do que é dito por Walker e Neeley (2004), em que, em uma relação B2B, o desempenho deve estar alinhado a expectativa.

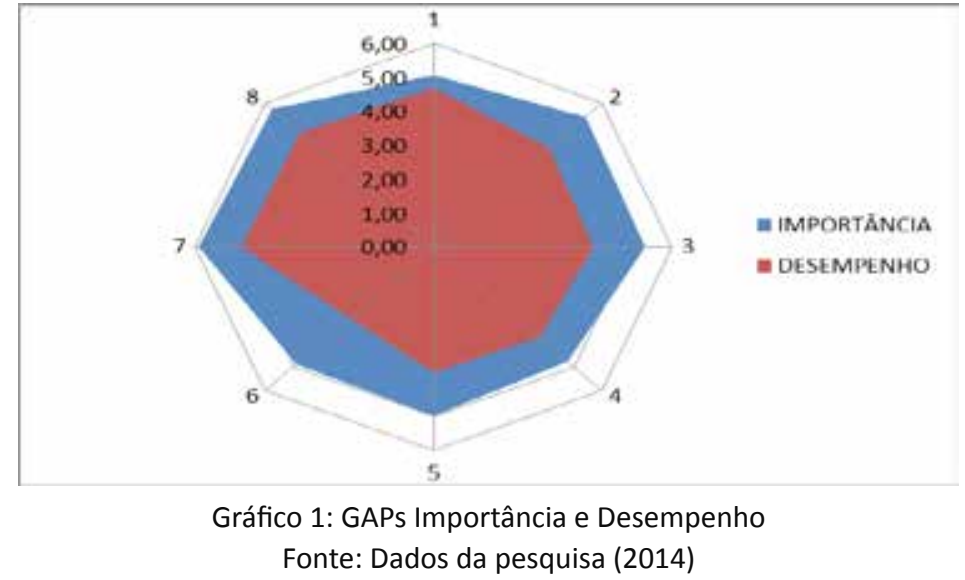

Para dar maior robustez ao estudo, optou-se por utilizar, também, o teste $t$ de Student, que, segundo Webster (2006), Stevenson (1981) e Triola (2005), teste que permite inferir sobre a igualdade de médias de amostras emparelhadas, sendo testadas para ver se o resultado é ou não zero. Verificou-se que a diferença entre as médias das duas amostras, é significativa. Para isso empregou-se um nível de confiança de $95 \%$ e nível de significância ( $\alpha$ ) de $5 \%$, para verificação da existência de diferenças estatisticamente comprovadas entre as médias. Por meio da análise do $p$-value do teste $t$ de Student, revela que existem diferenças estatisticamente significativas em todos os itens, visto que o $p$-value mostrou-se inferior a 0,05 em todos os testes. Tal resultado corrobora com os achados anteriores, provando que a importância e o desempenho de todos os itens são diferentes. Ademais, as correlações apresentadas na tabela, confirmam a baixa relação entre as variáveis, Importância e Desempenho, visto que o seu maior valor é referente à Imagem do Plano de Saúde, correspondendo a $57,14 \%$, indicando a falta de relação entre os construtos.

Diante das análises do GAP, do teste $t$-Student e das correlações, percebesse que a importância e o desempenho aparecem como elementos distintos e pouco relacionados. Tais análises são contrárias às preconizadas por Walker e Neeley (2004) e Day (2000) ao falar sobre o interesse em manter um bom relacionamento em um acordo $\mathrm{B} 2 \mathrm{~B}$, visto que os planos de saúde não tratam a satisfação baseada em valor como um processo de troca de valores, em que há a percepção de um valor que é dado e outro que é recebido. Desta forma, não existe o relacionamento baseado em valores esperados, fundamental para uma relação em longo prazo, necessária para uma boa parceria B2B.

Em relação à Matriz Importância e Desempenho (MARTILLA; JAMES, 1977), conforme a figura 2, abaixo, o eixo vertical representa a Importância, já o horizontal, o Desempenho. Slack, Chambers e Johnston (2009) apontam que não há um consenso na literatura para se dividir os 
quadrantes, porém, para este trabalho utilizou-se Almada e Tontini (2012), que diz que as setas que dividem os quadrantes são referentes às médias de cada eixo.

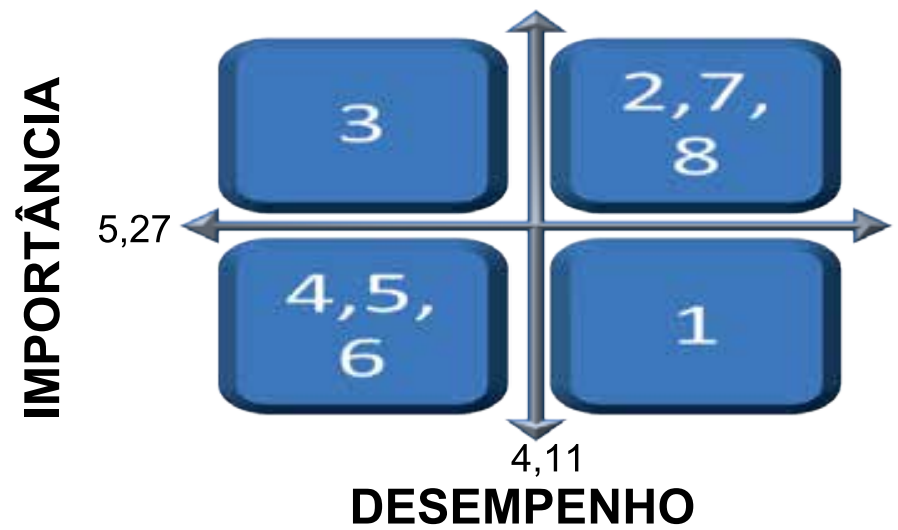

Figura: Matriz Importância x Desempenho.

Fonte: Próprios autores

O atributo localizado no quadrante $A$, representado pelo número 3 (Processos de autorizações para clientes), indica baixo desempenho e que se deve adequar urgentemente este item. Os processos de autorização para clientes são considerados extremamente importantes para os médicos, porém, não recebem a atenção devida dos planos de saúde, que devem se atentar a este item. Os itens localizados no quadrante $B$, indicado pelos números 2, 7 e 8 (Canal de comunicação com o plano de saúde; Cumprimento no prazo de pagamentos e Rede de prestadores de serviços, respectivamente), indicam grande vantagem competitiva, e devem ser mantidos pelos planos de saúde. $O$ quadrante $C$, apontado pelos números 4, 5 e 6 (Auditoria médica; Recurso de glosas nas contas médicas e Remuneração, respectivamente), representa itens que não tem bom desempenho, porém não possuem muita importância para o cliente. $O$ quadrante $D$, assinalado pelo número 1 (Imagem do plano de saúde), representa desperdício de recursos, pois o item possui grande desempenho, porém pouca importância. Dessa forma, é de se considerar a hipótese de reavaliar os investimentos nesses atributos, já que parte dos valores neles investidos poderia ser deslocada para outros itens de outros quadrantes (TONGE; MOORE, 2007).

Como apontam Carman (1990) e Cronin Jr e Taylor (1992) foram realizados os testes de GAPs e a matriz de Importância e Desempenho, que se completam. Para Matzler et al (2004), a importância pode variar conforme o desempenho, trazendo um maior grau de complexidade na análise das oportunidades de melhoria. Assim, a análise dos GAP's traz uma perspectiva diferente para a análise de oportunidades de melhoria, por utilizar outro aspecto. Considerando a análise de desempenho, parece ser evidente a necessidade de ação para que se eleve a percepção de desempenho dos critérios pior avaliados, porém, ao considerarmos a distância entre a importância de um atributo e seu desempenho - o que é denominado GAP de desempenho e conforme Parasuraman, Zeithaml e Berry (1985, 1988, 1990); Gianesi e Corrêa (2008) - essa discrepância entre o percebido e o esperado que precisa ser tratada prioritariamente. Assim, do ponto de vista gerencial, maiores GAP's negativos e maior importância, geram efeitos mais danosos na satisfação dos médicos credenciados. Com GAP's menores em fatores de menor importância, o efeito pode ser mínimo no grau de satisfação.

Os GAP's encontrados na pesquisa demonstram, conforme apontam Parasuraman, Zeithaml e Berry (1985, 1988, 1990) e Gianese e Corrêa (2008), uma falha de qualidade entre todos os itens pesquisados, o que influi negativamente na satisfação dos médicos em relação aos pla- 
nos de saúde. Dentre todos os elementos, o maior GAP encontrado foi o que diz respeito à remuneração, sendo sua Importância com média de 4,89 e desempenho de 2,97, tendo o GAP de 1,91. Isto indica que mesmo que a remuneração seja um elemento considerado pelos entrevistados como sendo abaixo da média, considerando a importância, os planos de saúde desempenham um papel muito ruim para este aspecto. Os elementos encontrados no quadrante B (Canal de comunicação com o plano de saúde; Cumprimento no prazo de pagamentos e Rede de prestadores de serviços, respectivamente), também devem ser analisados levando em consideração o GAP encontrado, o que representa vantagem competitiva pela Matriz, na realidade não representam, quando comparados à análise dos GAPs.

\section{CONSIDERAÇÕES FINAIS}

Tendo em vista o grande crescimento de adeptos aos planos de saúde no Brasil, e a carência de estudos quando se fala sobre a satisfação do médico perante o plano de saúde, o presente artigo teve como objetivo identificar os principais elementos que contribuem para a satisfação do médico credenciado ao plano de saúde e como é percebido o desempenho destes elementos pelos planos de saúde, para tanto utilizou abordagem qualitativa em um primeiro momento e uma abordagem quantitativa posteriormente. Os elementos foram identificados com base em um grupo focal e apoiados pela literatura especializada (SILVA, 2003; LIBERALESSO, 2011). Após os elementos serem previamente identificados, um questionário foi formulado e enviado para os médicos cadastrados no banco de dados do CRM-ES.

A fim de responder ao problema de pesquisa, o artigo utilizou a Matriz Importância e Desempenho (MARTILLA; JAMES, 1977) e análise dos GAPs de qualidade (GIANESE; CORRÊA, 2008), e para dar robustez aos resultados, bem como comprovar sua validade estatística, utilizouse análise do teste t-student e análise de correlação, vistos em Levine (2012). Em linhas gerais, com as análises feitas, confirmou-se que o desempenho foi abaixo do esperado para todos os quesitos pesquisados, visto que em todas as variáveis ele é menor que a importância. Tal fato foi demonstrado pela análise dos GAPs de qualidade e comprovados pelos testes de correlação e t-student. Resultado este que indica que os médicos não estão satisfeitos com nenhum dos quesitos, que devem ser melhorados pelos planos de saúde.

Todos os itens demonstraram desempenho inferior ao esperado, porém, ao se analisar a Matriz de Importância e Desempenho, observa-se que alguns itens estão em áreas críticas, como o item "processo de autorização para clientes", que é considerado extremamente importante para os médicos, porém são desempenhados de forma muito pouco satisfatória pelos planos de saúde, exigindo investimentos prioritários neste quesito. Em contra partida, os elementos "Auditoria médica; Recurso de glosas nas contas médicas e Remuneração", mesmo considerados não muito bem desempenhados pelos planos de saúde, não são itens considerados tão importantes, em relação aos outros elementos pesquisados, o que não é visto de forma tão prioritária para se realizar melhorias. Quando se fala da imagem do plano de saúde perante a sociedade, este quesito é bem desempenhado pelos planos de saúde, porém não é considerado importante pelos médicos, concluindo então que há um desperdício de recursos para este elemento, quando se fala da satisfação do médico.

Walker e Neeley (2004) e Gummerson (1996) apontam que um relacionamento B2B bem sucedido é baseado nas trocas de valores, em que ambas as partes estão satisfeitas e enxergam o desempenho da outra parte como compatível ao esperado. Gummerson (1996) aponta que nos casos em que uma das partes não está satisfeita, a relação B2B tende ao insucesso e ao 
declínio, o que não foi identificado no presente estudo, em que os respondentes demonstram insatisfação em todos os itens, enxergando desempenho abaixo da expectativa. Os resultados destoam do que é encontrado na literatura, pois as associações entre médicos e planos de saúde, conforme indicam os dados da ANS (2013) estão cada vez mais frequentes.

A relação entre o crescente número de associações entre médicos e planos de saúde, não se explica pela relação entre importância e desempenho dos itens levantados. É característica de um ambiente complexo, como a relação entre planos de saúde e médicos, a difícil identificação de como estão articuladas as relações dos atores (FRANCE; GROVER, 1992), sendo identificado no presente estudo, que as relações de parceria não são consolidadas levando em consideração a satisfação, confirmando o que é apontado por Silva (2003).

O estudo confirma o que é indicado por Silva (2003) e Liberalesso (2011) em que os médicos estão reféns dos planos de saúde, dependendo deles para exercerem sua atividade profissional, em que, mesmo considerando seu desempenho inferior ao esperado, ainda permanecem associados a eles.

O presente artigo respondeu ao problema de pesquisa, pois por meio do grupo focal, identificou os principais elementos que contribuem para a satisfação do médico credenciado ao plano de saúde, e, através da análise dos questionários enviados identificou como o médico percebe o desempenho destes elementos pelos planos de saúde.

O artigo tem como limitação o fato de a Matriz de Importância e Desempenho identificar alguns atributos como vantagem competitiva, o que não acontece, pois todos os itens possuem o desempenho menor do que a importância observada. Outra limitação do artigo se dá pela não diferenciação de cada um dos planos de saúde, sendo feita uma análise geral dos planos, deste modo, resultados diferentes podem ser encontrados ao se trabalhar com um plano especifico. Sugere-se para pesquisas futuras uma análise comparativa entre planos de saúde para um estudo mais detalhado a respeito da satisfação do médico em relação aos planos de saúde.

\section{REFERÊNCIAS}

AGÊNCIA NACIONAL DE SAÚDE SUPLEMENTAR. Caderno de informação da Saúde Suplementar: beneficiários, operadoras e planos. Dezembro, 2013.

ALMADA, I. W.; TONTINI, G. Atributos críticos de satisfação em serviços de arquitetura: visão do cliente $\times$ visão do arquiteto. Revista Produção, v.22, n.2, pp. 1-12. Abr./Jun. 2012.

BOGDAN, R.; BIKLEN, S. Características da investigação qualitativa. In: Investigação qualitativa em educação: uma introdução à teoria e aos métodos. Porto, Porto Editora, p.47-51, 1994.

CARMAN, James M. Consumer Perceptions of Service Quality: an assessment of the
SERVQUAL dimensions. Journal of Marketing, New York: American Marketing Association, 1990. v. 66(1), p. 33-35.

CRONIN JR, J. Joseph,;TAYLOR, A Steven. Measuring Service Quality: a reexamination and a extension. Journal of Marketing, New York: American Marketing Association, Jul. 1992. v.56, p. 55-68.

CHANG, S-H.; WANG, K-Y.; CHIH, W-H.; TSAI, $\mathrm{W}-\mathrm{H}$. Building customer commitment in business-to-business markets. Industrial Marketing Management, v. 41, n. 6, p. 940950, 2012.

CHURCHILL JR., G. A. Marketing research: methodological foundations. Sixth Edition. Orlando: Dryden Press, 1995.

; SURPRENANT, C. An investigation into the determinants of customer satisfaction. 
Journal of Marketing Research, v. 19, p. 491504, 1982.

CURI, D. P. Orientação para o mercado: a relação entre orientação para a tecnologia e o marketing relacional. 2007. 228 f. Tese (Doutorado em Engenharia de Produção) Escola Politécnica, Universidade de São Paulo, São Paulo, 2007.

DAY, S. Managing market relationships. Journal of the Academy of Marketing Science, v. 28, n. 1, p. 24-30, 2000.

DALL'AGNOL, Clarice Maria; TRENCH, Maria Helena. Grupos focais como estratégias metodológicas em pesquisas na enfermagem. Revista Gaúcha de Enfermagem, Porto Alegre, v. 20, n. 1, p. 5-25, jan. 1999.

FLICK, Uwe. Uma introdução à pesquisa qualitativa. 2. ed. Porto Alegre: Bookman, 2004.

FRANCE, R.; GROVER, R. What is the Care Product? Journal of Health Care Marketing, Junho, 1992.

GIANESI, I.; CORRÊA, H. Administração estratégica de serviços: operações para a satisfação do cliente. São Paulo: Atlas, 2008.

GARVER, M. S. Best practices in identifying customer-driven improvement opportunities. Industrial Marketing Management, [s.I], v. 32, n. 6, p. 455-466, Aug. 2003.

GOMES, J.; SCHUCH JR., V; KNEIPP, J.; ROSA, L.; BICHUETI, R. Satisfação dos serviços: Uma análise da percepção dos clientes do Banco do Brasil. Rev. Adm. UFSM, Santa Maria, v. 5, n. 1, p. 126-144, jan./abril. 2012.

GUMMERSSON, E. Relationship marketing and imaginary organizations: a synthesis. European Journal of Marketing, West Yorkshire, v. 30, n. 3, p. 31-34, 1996.

HAIR, J. F., JR.; BUSH, R. P.; ORTINAU, D. $J$. Marketing research: a practical approach for the new millennium. Boston : Irwin/McGrawHill, 2000.

HENNING-THURAU, T; KLEE, A. The Impact of Customer Satisfaction and Relationship Quality on Customer Retention: A Critical Reassessment and Model Development. Psychology \& Marketing, 1997.

KINNEAR, T.; TAYLOR, J. R. Marketing Research: an applied approach. 5. Ed. New York: McGrawHill, 1996.

LACERDA, T.; MEDONÇA, B. Marketing B2B: Mapeamento dos trabalhos acadêmicos no Brasil de 1998 a 2007. Rev. Adm. UFSM, Santa Maria, v. 3, n. 2, p. 219-229, mai./ago. 2010.

LEAL, R. M.; MATOS. J. B. B. Planos de saúde: uma análise dos custos assistenciais e seus componentes. Revista de Administração de Empresas, v. 49, n.4, 2009.

LEVINE, D. M.; STEPHAN, D.; KREHBIEL, T. C.; BERENSON, M. L. Estatística - Teoria e Aplicações: usando o Microsoft Excel em português. 6. Ed. Rio de Janeiro: LTC, 2012.

LIBERALESSO, Paulo. Cidadão padesse entre SUS e plano de saúde. Correio Brasiliense. 24. jun. 2011. Medicina.

MALHOTRA, N. K. Pesquisa de Marketing. 3.ed. Porto Alegre: Bookman, 2006.

MARTILLA, J. A.; JAMES, J. C. Importanceperformance analysis. Journal of Marketing, New York, v, 41, n. 1, p.77 - 79, 1977.

MATZLER, K.; BAILOMB, F.; HINTERHUBER, H.; RENZLA, B.; PICHLERB, J. The asymmetric relationship between atribute-level performance and overall customer satisfaction: a reconsideration of the importanceperformance analysis. Industrial Marketing Management, [s.1], v. 33, n.4, p. 271-277, 2004.

McKENNA, Regis. Marketing de Relacionamento. Rio de Janeiro: Campus, 1996. 
MORAN, M. R.; SILVA, M. A.; RAMALHO, N. C.; GOUVÊA, M. A. Satisfação de compradores e posicionamento estratégico no B2B: uma proposta de aplicação de resultados de Pesquisa na formulação de estratégias. Latin America Journal of business management. v. 4, n. 2, p. 81-104, jul-dez/2013, Taubaté, SP, Brasil.

MORGAN, R.; HUNT, S. The Commitment-Trust Theory of Relationship Marketing. Journal of Marketing, v. 58, p. 20-38, July, 1994.

OLIVER, R. L. A cognitive model of the antecedents and consequences of satisfaction decisions. Journal of Marketing Research, v. 17, p. 460-469, 1980.

OLKOSKI, G.; USTER, R.; MARQUES, L.; SILVA, J. Marketing de Relacionamento e Software de CRM: Um estudo de caso em uma concessionária de automóveis. Rev. Adm. UFSM, Santa Maria, v. 2, n. 3, p. 417-432, set./ dez. 2009.

PALMATIER, R. W.; DANT, R. P.; GREWAL, D.; EVANS, K. R. Factors influencing the effectiveness of relationship marketing: a meta-analysis. Journal of Marketing, v. 70, out. 2006.

PARASURAMAN, A.; ZEITHAML, V. A.; BERRY, L. L., Delivering quality service. Balancing customer perceptions and expectations. New York: The Free Press, 1990.

multiple item scale for measuring consumer perceptions of service quality. Journal of Retailing, v. 64, n. 1, p. 12- 40, 1988.

A conceptual

model of services quality and its implication for future research. Journal of Marketing, $v$. 49, n. 4, p. 41-50, 1985.

PINHO, J. Redes e capital social no domínio do marketing de relacionamento. Uma análise de possíveis complementaridades. Revista portuguesa e brasileira de gestão. Vol. 12, n.4. Lisboa, dez. 2013
POPPER, Karl. A lógica da pesquisa científica. 2. ed. São Paulo: Cultrix, 1972.

PRIETO, V. C.; CARVALHO. M. M. Gestão do relacionamento com o cliente em um mercado Business to business. Revista produção online. V.05, n.01, 2005.

RICHARDSON, L. J. Pesquisa social: métodos e técnicas. São Paulo: Atlas, 2007.

ROSSI, C. A. V.; SLONGO, L. A. Pesquisa de satisfação de clientes: o estado-da-arte e proposição de um método brasileiro. Revista de Administração Contemporânea, v.2, n.1. Curitiba, 1998.

ROZZET, K.; DEMO, J. Desenvolvimento e validação fatorial da escala de relacionamento com os clientes (ERC). Revista de Administração de Empresas, vol. 50, n.04, são Paulo, 2010.

SATO, F. R. L. A teoria da agência no setor da saúde: o caso do relacionamento da Agência Nacional de Saúde Suplementar com as operadoras de plano de assistência supletiva no Brasil. Revista de Administração Pública, v.41, n.1. Rio de Janeiro, 2007.

SIQUEIRA, M. M. M. (Org.). Medidas do comportamento organizacional: ferramentas de diagnóstico e de gestão. Colaboração: Álvaro Tamayo et al. Porto Alegre: Artmed, 2008.

SILVA, A. A. Relação entre Operadoras de Planos de Saúde e Prestadores de Serviços Um Novo Relacionamento Estratégico. Agência Nacional de Saúde Suplementar. Porto Alegre, 2003.

SLACK, N.; CHAMBERS, S; JOHNSTON, R. Administração da Produção. Tradução Henrique Luiz Corrêa. 3. Ed. São Paulo: Atlas, 2009.

SMITH, J.K. Quantitative vs. Qualitative Research: An Attempt to Clarify the Issue. Educational Researcher, Vol. 12, N. 03; Mar. 1983. 
STEVENSON, W. J. Estatística aplicada à administração. São Paulo: Harper \& Row do Brasil, 1981.

TONGE, Joanna; MOORE, Susan A. Importancesatisfaction analysis for marine-park hinterlands: A Western Australia case study. Tourism Management, v.28, p. 768-776, 2007. TONTINI, G.; SANT'ANA, A. J. Identificação de atributos críticos de satisfação em um serviço através da análise competitiva do gap de melhoria. Gestão e Produção, São Carlos, v.14, n.1, p.43-54, 2007.

TRIOLA, M. F. Introdução à Estatística. Rio de Janeiro: LTC, 2005.

WALKER, L. H.; NEELEY, S. E. Customer relationship building on the internet in $b 2 b$ marketing: a proposed typology. Journal of Marketing Theory and Practice, 19-35, 2004.

WEBSTER. A. L. Estatística aplicada à administração e economia. Trad. Maria Cecília Sonoe Oliva, Helena Maria Ávila de Castro. São Paulo: McGraw-Hill, 2006.

WEICK, K. E. Sensemaking in organizations. Thousand Oaks, Sage, p. 231, 1995.

WEITZ, Barton A.; BRADFORD, Kevin D. Personal selling and sales management: a relationship marketing perspective. Journal of Academy of Marketing Science, v. 27, n. 2, 1999. 